\title{
Rattling Transport of Lithium Ion in the Cavities of Model Solid Electrolyte Interphase
}

\author{
T Aashish ${ }^{\dagger}$ and Bhabani S. Mallik ${ }^{\dagger, *}$ \\ ${ }^{\dagger}$ Department of Chemistry, Indian Institute of Technology Hyderabad, Kandi-502 285, Sangareddy, Telangana, India
}

\section{Choice of force field parameters and other simulation details:}

LMC system was simulated using both scaled and unscaled charges for our comparative study on prospective force fields. We created four sets of simulations: OPLS-scaled, OPLS-unscaled, GAFF-scaled, and GAFF-unscaled. We scaled the charges to reduce the electrostatic interactions of the molecules to $80 \% 1$. Then we annealed the searing LMC components steadily to the required temperatures for our study. We decided to anneal the heated systems to three different temperatures: 293 , 373, and $473 \mathrm{~K}$. After cooling, the particles were set to run for equilibration in the isobaric-isothermal $(N p T)$ ensemble conditions for a total of $15 \mathrm{~ns}$, the first $5 \mathrm{~ns}$ using Berendsen ${ }^{2}$ pressure coupling and the remaining $10 \mathrm{~ns}$ by using ParrinelloRahman $^{3}$ Barostat at a constant pressure of 1 bar. We employed the V- Rescale ${ }^{4}$ thermostat to control the temperature. Then we equilibrated the systems in the canonical ensemble (NVT) for $20 \mathrm{~ns}$, followed by a final long run of $100 \mathrm{~ns}$ in microcanonical (NVE) ensemble. After applying this procedure to the four sets of simulation runs on LMC, the properties and results were compared. We employed the same set of simulation runs in their respective ensembles for other carbonates as well. The Mean squared displacements for LMC depicted in Figure $\mathrm{S} 1$ show that the $\mathrm{Li}^{+}$ions displace to a greater extent in simulations for force fields incorporating scaled charges than for force fields with no scaling. Such a result comes as no surprise as the electrostatic forces become weaker on account of charge scaling. Here, we tested both the force fields on one of our systems to determine the more suitable of the two.

We simulated a system containing 200 particles in $N p T$ equilibration conditions for $100 \mathrm{~ns}$, first using Berendsen Barostat for $10 \mathrm{~ns}$ and later with Parinello-Rahman Barostat, at $293 \mathrm{~K}$. At first, equilibration was done on the carbonates at a pressure of 1 bar using simple isotropic pressure-coupling. Next, we increased the pressure to 10 bar using semi-isotropic pressure coupling along the z-direction. We performed subsequent simulations by increasing the magnitude of stress experienced by the simulation box. We kept on raising the pressure by 10 bar for the proceeding simulations. The average box length (along the z-direction) computed from the system at 1 bar pressure was taken to be ' $l_{o}$ ', and we labeled the box lengths calculated from remaining simulations as ' $l_{i}$ '. The box lengths were calculated in the time interval when the fluctuation of the values of density is less and around the average value. We calculated the strain suffered by the simulation box using the unperturbed value of ' $l_{o}$ ' and the respective ' $l_{i}$ ' value for that simulation box. The average stress applied along $\mathrm{z}$ direction was plotted against the strain, and the value of the Young's modulus was estimated from the plot.

\section{References}

(1) Muralidharan, A.; Chaudhari, M. I.; Pratt, L. R.; Rempe, S. B. Molecular Dynamics of Lithium Ion Transport in a Model Solid Electrolyte Interphase. Sci. Rep. 2018, 8, 1-8.

(2) Berendsen, H. J. C.; Postma, J. P. M.; van Gunsteren, W. F.; DiNola, A.; Haak, J. R. Molecular Dynamics with Coupling to an External Bath. J. Chem. Phys. 1984, 81, 3684-3690.

(3) Parrinello, M.; Rahman, A. Polymorphic Transitions in Single Crystals: A New Molecular Dynamics Method. J. Appl. Phys. 1981, 52, 7182-7190.

(4) Bussi, G.; Donadio, D.; Parrinello, M. Canonical Sampling through Velocity Rescaling. J. Chem. Phys. 2007, 126, 014101. 
Table S1. Total no. of anions and cations taken in the simulation box, simulation box lengths at various temperatures and the Diffusion coefficients for lithium ions at 293,373 and $423 \mathrm{~K}$ for all the three systems.

\begin{tabular}{|c|c|c|c|c|c|}
\hline S.no & SYSTEM & No. of Cations & No. of Anions & \multicolumn{2}{|c|}{$\begin{array}{l}\text { Diffusion coefficients of } \\
\text { Lithium ion }\left[\mathrm{m}^{2} \mathrm{~s}^{-1}\right]\end{array}$} \\
\hline \multirow{3}{*}{1.} & \multirow{3}{*}{$\begin{array}{l}\text { Lithium Methylcarbonate } \\
\text { (LMC) }\end{array}$} & \multirow{3}{*}{500} & \multirow{3}{*}{500} & $293 \mathrm{~K}$ & $1.0 \times 10-13$ \\
\hline & & & & $373 K$ & $5.0 \times 10-13$ \\
\hline & & & & $423 \mathrm{~K}$ & $8.0 \times 10-12$ \\
\hline \multirow[t]{3}{*}{2.} & \multirow{3}{*}{$\begin{array}{l}\text { Lithium Ethylcarbonate } \\
\text { (LEC) }\end{array}$} & \multirow{3}{*}{500} & \multirow{3}{*}{500} & $293 \mathrm{~K}$ & $1.0 \times 10-13$ \\
\hline & & & & $373 \mathrm{~K}$ & $3.0 \times 10-13$ \\
\hline & & & & $423 \mathrm{~K}$ & $3.8 \times 10-12$ \\
\hline \multirow{3}{*}{3.} & \multirow{3}{*}{$\begin{array}{l}\text { Lithium Butylcarbonate } \\
\text { (LBC) }\end{array}$} & \multirow{3}{*}{500} & \multirow{3}{*}{500} & $293 K$ & $2.0 \times 10-13$ \\
\hline & & & & $373 \mathrm{~K}$ & $3.0 \times 10-13$ \\
\hline & & & & $423 \mathrm{~K}$ & $2.8 \times 10-12$ \\
\hline
\end{tabular}


Table S2. Non-bonding force field parameters for LMC, LEC and LBC. Charges were scaled to 0.80

\begin{tabular}{|c|c|c|c|c|}
\hline System & Atom & Charge $q(e)$ & $\sigma(\AA)$ & $\varepsilon$ (kJmol-1) \\
\hline \multirow[t]{8}{*}{ LMC } & 02 & -0.6366 & 2.96 & 0.8786 \\
\hline & $\mathrm{C} 1$ & 0.8261 & 3.40 & 0.3598 \\
\hline & $\mathrm{O3}$ & -0.6606 & 2.96 & 0.8786 \\
\hline & 01 & -0.4225 & 3.00 & 0.7113 \\
\hline & $\mathrm{C} 2$ & 0.1224 & 3.40 & 0.4577 \\
\hline & $\mathrm{H} 1$ & -0.0019 & 2.47 & 0.0657 \\
\hline & $\mathrm{H} 2$ & -0.0250 & 2.47 & 0.0657 \\
\hline & $\mathrm{H} 3$ & -0.0020 & 2.47 & 0.0657 \\
\hline \multirow[t]{11}{*}{ LEC } & $\mathrm{C} 1$ & -0.3205 & 3.40 & 0.4577 \\
\hline & $\mathrm{H} 1$ & 0.0676 & 2.65 & 0.0657 \\
\hline & $\mathrm{H} 2$ & 0.0224 & 2.65 & 0.0657 \\
\hline & $\mathrm{H} 3$ & 0.0676 & 2.65 & 0.0657 \\
\hline & $\mathrm{C} 2$ & 0.4499 & 3.40 & 0.4577 \\
\hline & $\mathrm{H} 4$ & -0.0575 & 2.47 & 0.0657 \\
\hline & $\mathrm{H} 5$ & -0.0575 & 2.47 & 0.0657 \\
\hline & 01 & -0.4922 & 3.00 & 0.7113 \\
\hline & $\mathrm{C3}$ & 0.8144 & 3.40 & 0.3598 \\
\hline & 03 & -0.6522 & 2.96 & 0.8786 \\
\hline & $\mathrm{O} 2$ & -0.6420 & 2.96 & 0.8786 \\
\hline \multirow[t]{17}{*}{ LBC } & $\mathrm{C} 1$ & -0.0807 & 3.40 & 0.4577 \\
\hline & $\mathrm{H} 1$ & -0.0036 & 2.65 & 0.0657 \\
\hline & $\mathrm{H} 2$ & 0.0019 & 2.65 & 0.0657 \\
\hline & $\mathrm{H} 3$ & 0.0019 & 2.65 & 0.0657 \\
\hline & $\mathrm{C} 2$ & 0.1700 & 3.40 & 0.4577 \\
\hline & $\mathrm{H} 4$ & -0.0552 & 2.65 & 0.0657 \\
\hline & $\mathrm{H} 5$ & -0.0553 & 2.65 & 0.0657 \\
\hline & $\mathrm{C3}$ & -0.0962 & 3.40 & 0.4577 \\
\hline & $\mathrm{H} 6$ & 0.0144 & 2.65 & 0.0657 \\
\hline & $\mathrm{H} 7$ & 0.0145 & 2.65 & 0.0657 \\
\hline & C4 & 0.3104 & 3.40 & 0.4577 \\
\hline & $\mathrm{H} 8$ & -0.0309 & 2.47 & 0.0657 \\
\hline & $\mathrm{H} 9$ & -0.0311 & 2.47 & 0.0657 \\
\hline & 01 & -0.4967 & 3.00 & 0.7113 \\
\hline & $\mathrm{C} 5$ & 0.8385 & 3.40 & 0.4577 \\
\hline & 03 & -0.6559 & 2.96 & 0.8786 \\
\hline & $\mathrm{O} 2$ & -0.6461 & 2.96 & 0.8786 \\
\hline
\end{tabular}


Table S3. The table consists of the box length values taken for each of the systems. The densities calculated from the isothermal-isobaric simulation runs at three temperatures are given.

\begin{tabular}{|c|c|c|c|}
\hline System & Box Length [nm] & Temperature $[\mathrm{K}]$ & Density $[\mathrm{gcm}-3]$ \\
\hline \multirow{3}{*}{ LMC } & \multirow[t]{3}{*}{3.7} & 293 & 1.436 \\
\hline & & 373 & 1.431 \\
\hline & & 423 & 1.408 \\
\hline \multirow{3}{*}{ LEC } & \multirow[t]{3}{*}{4.1} & 293 & 1.264 \\
\hline & & 373 & 1.250 \\
\hline & & 423 & 1.227 \\
\hline \multirow{3}{*}{ LBC } & \multirow[t]{3}{*}{4.7} & 293 & 1.133 \\
\hline & & 373 & 1.097 \\
\hline & & 423 & 1.061 \\
\hline
\end{tabular}




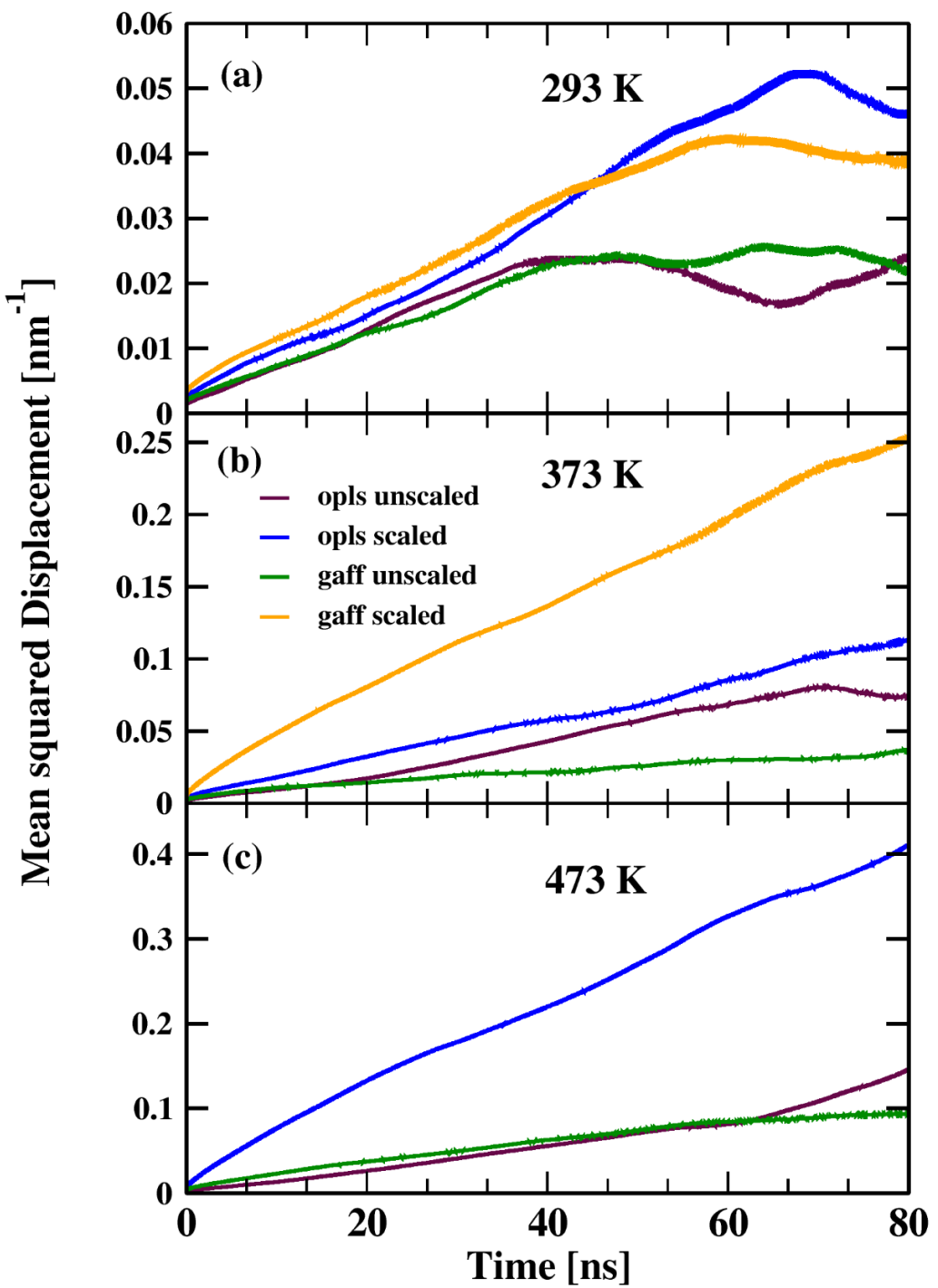

Figure S1. The figure represents the mean squared displacement curves for lithium ions in LMC. The figure compares the displacements for the four force fields at three temperatures of $293 \mathrm{~K}, 393 \mathrm{~K}$ ad $423 \mathrm{~K}$. 


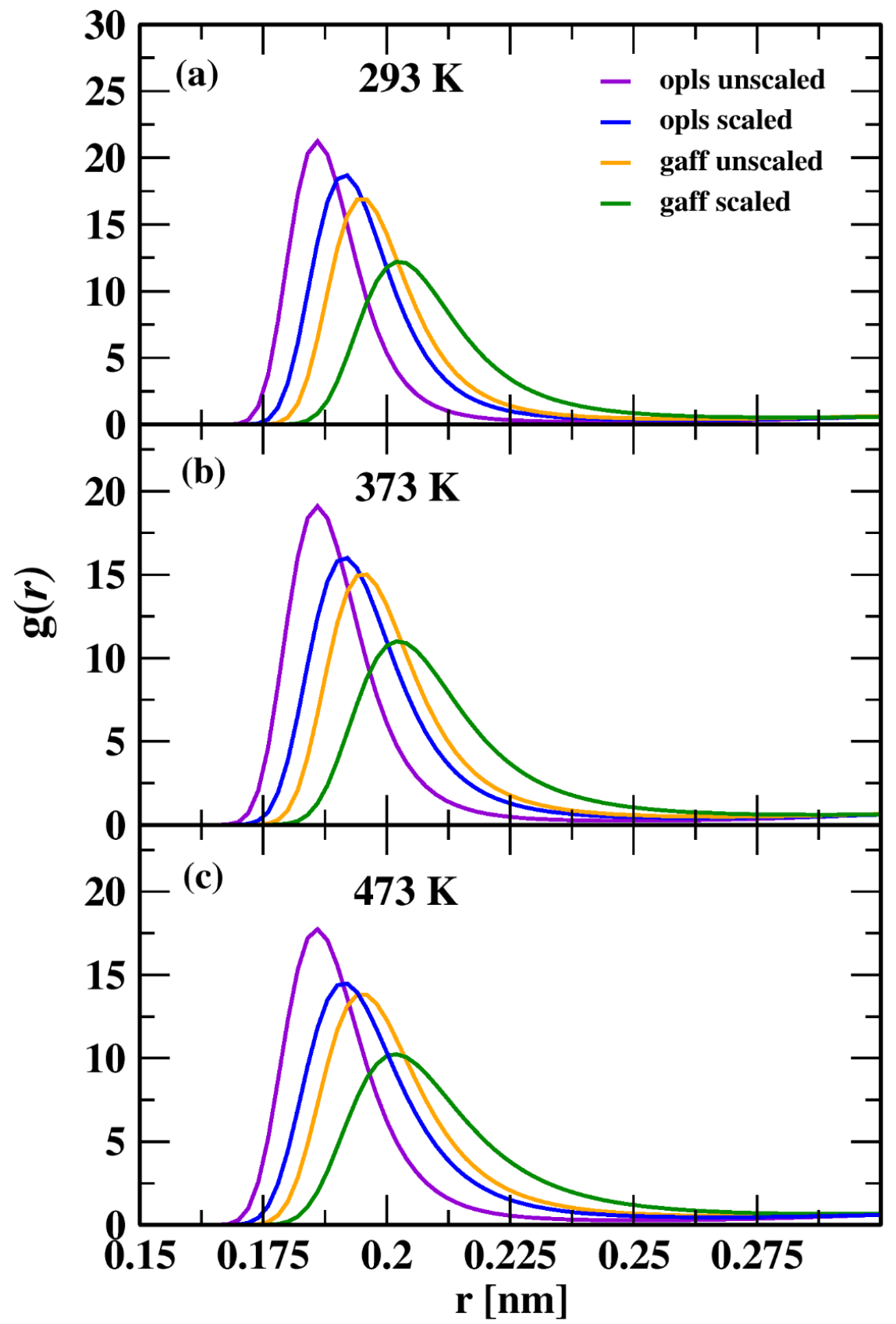

Figure S2. Radial Distribution curves for Lithium methyl Carbonate using four force fields, OPLS-unscaled, OPLSscaled, GAFF-unscaled and GAFF-scaled. 

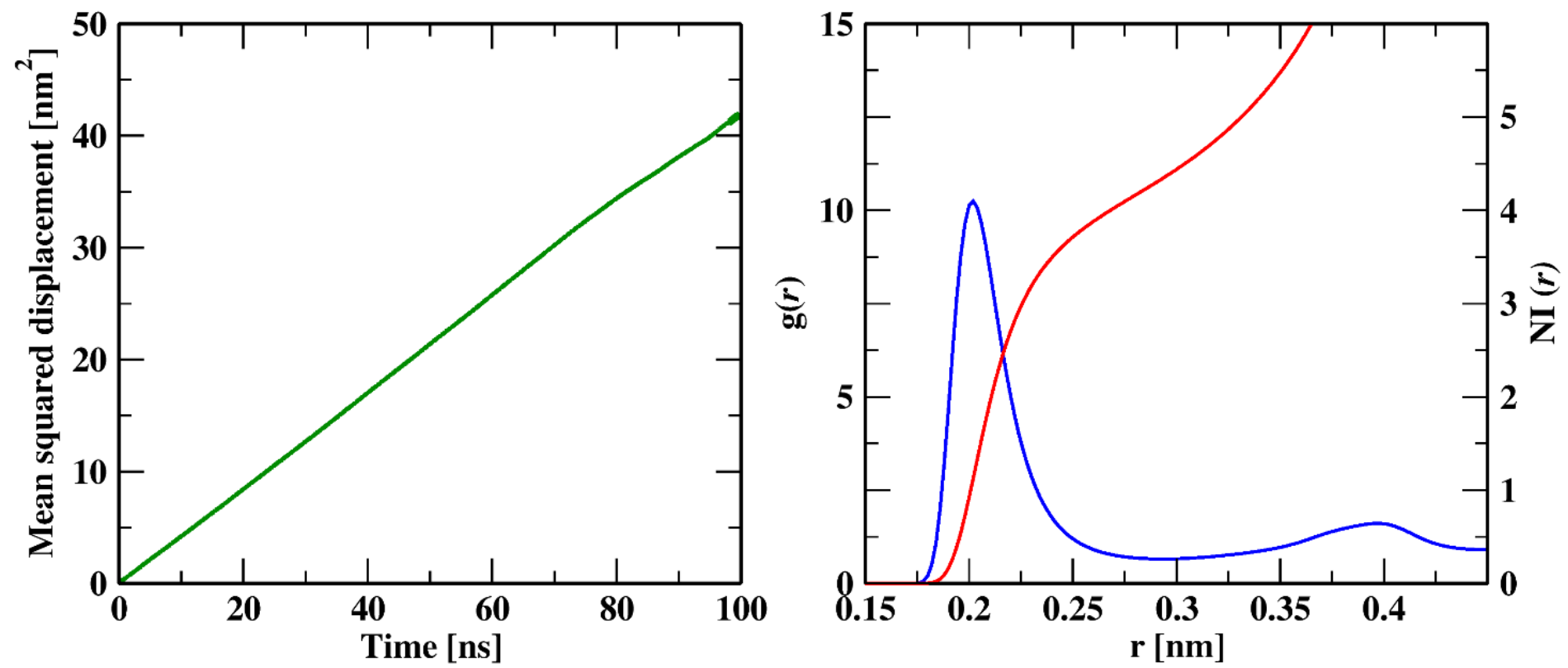

Figure S3. The Mean squared displacement curve for Lithium Methyl Carbonate at 473K using GAFF-scaled force field and the Radial Distribution plot along with Number Integral curve at 473K. During the analysis of the data, we found that at $473 \mathrm{~K}$, simulation from GAFF scaled force field showed a dramatic upsurge in MSD values, compared to simulations with other force fields at this temperature. The mean squared displacement achieved by $\mathrm{Li}^{+}$ ions was around $42 \mathrm{~nm}^{2}$ at the end of the simulation run, which is much larger than the displacement values from all other force fields combined. 


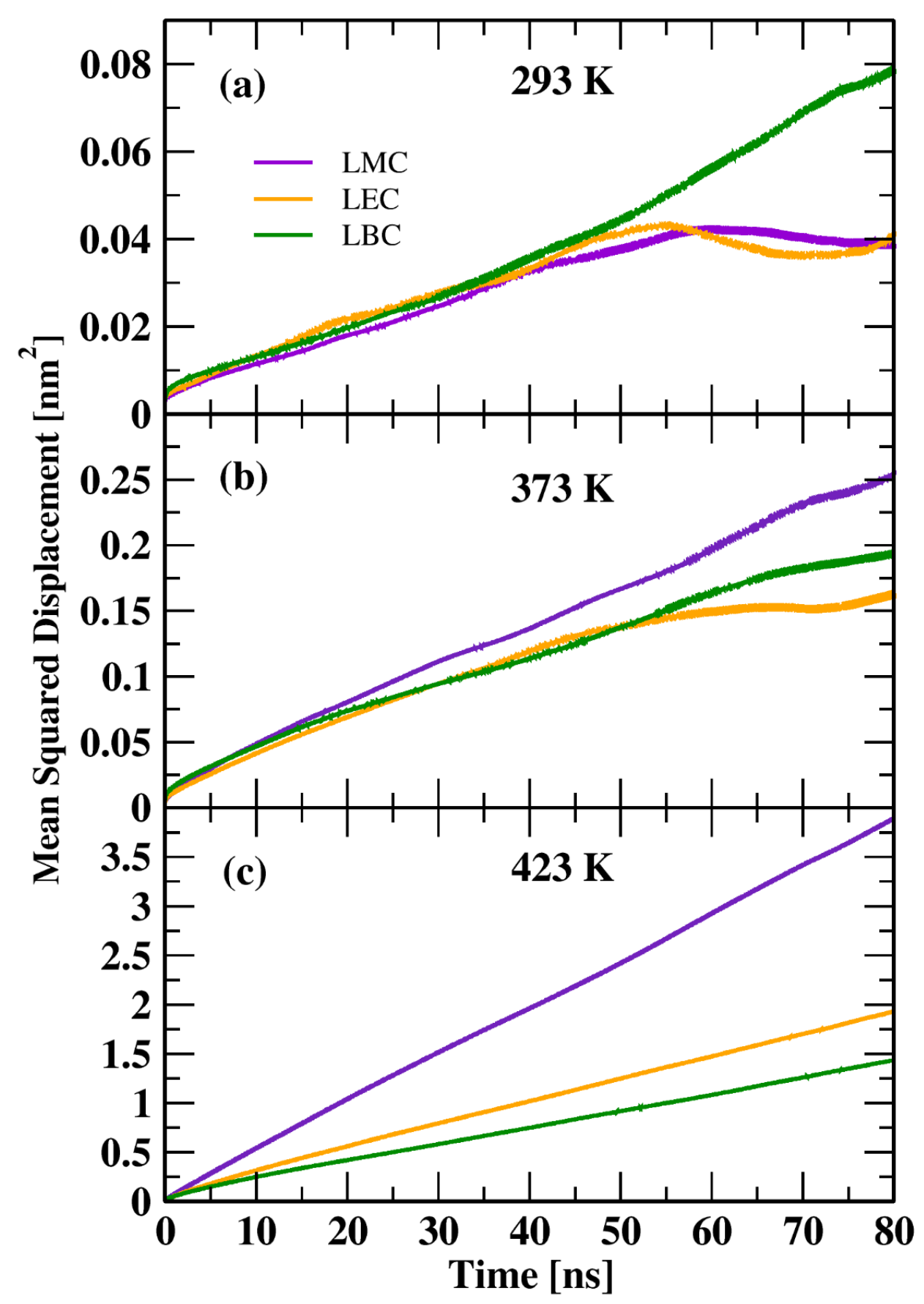

Figure S4. Mean squared Displacement plots for Lithium Methyl Carbonate (LMC), Lithium Ethyl Carbonate (LEC) and Lithium Butyl Carbonate (LBC) depicting the motion of lithium ions at three different temperatures of 293K, 373K and 423K. This figure shows the MSD curves for the three carbonates. At $293 \mathrm{~K}$, the trapping regions are large while the diffusive domains are small. The $\mathrm{Li}^{+}$ions were found to be trapped between the carbonate molecules and firmly bound to the oxygen atoms. The low thermal energy is insufficient to break the strong coordination between the cations and anions. At higher temperatures, we find that the curves become approximately linear, and the trapping zones are hard to figure out. 


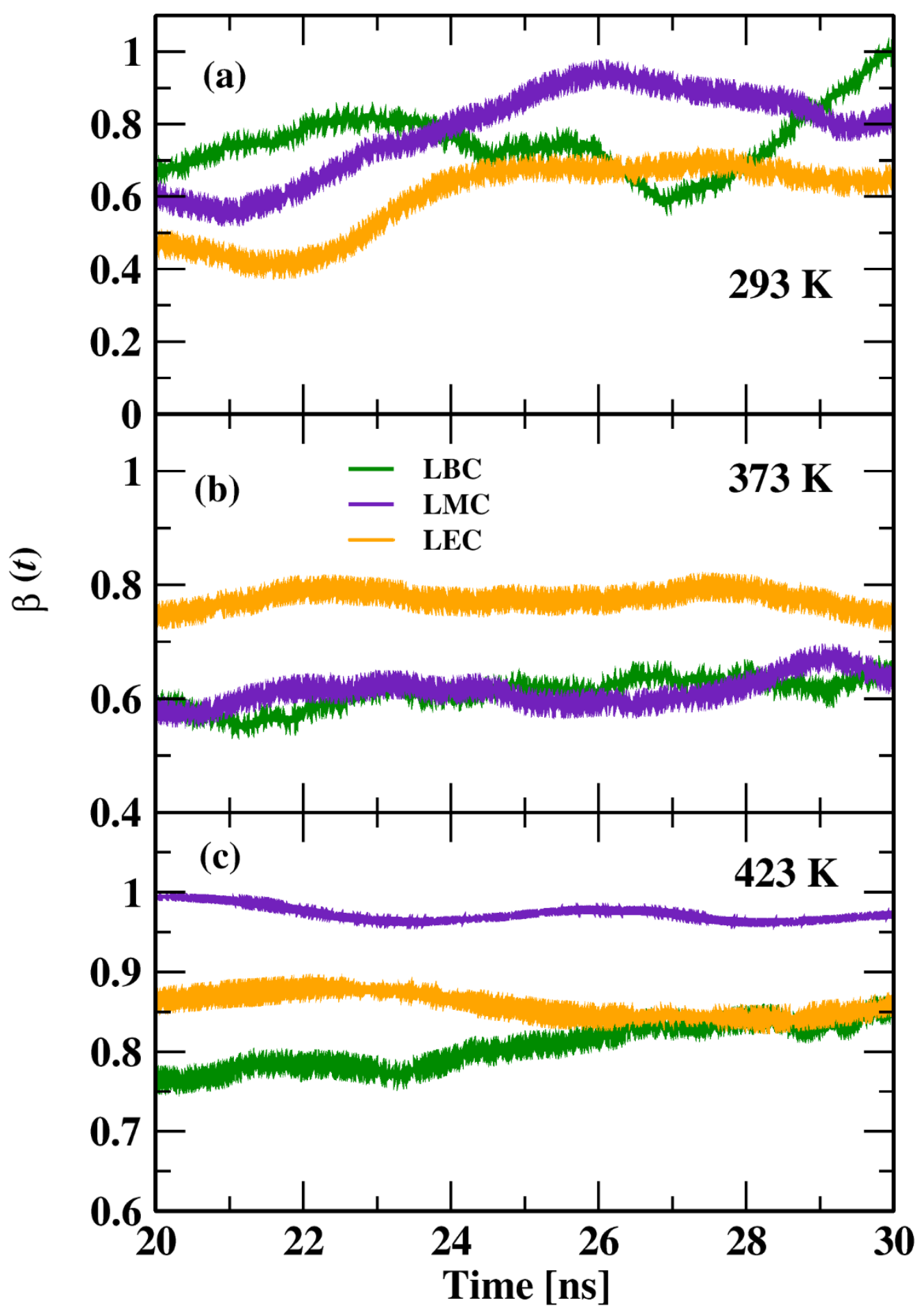

Figure S5. The figures represent the Beta plots calculated from the data obtained by mean squared displacement of the lithium ions from 20 to 30 ns for Lithium Methyl Carbonate (LMC), Lithium Ethyl Carbonate (LEC) and Lithium Butyl Carbonate (LBC) at three temperature values 293K, 373K and $423 \mathrm{~K}$. 


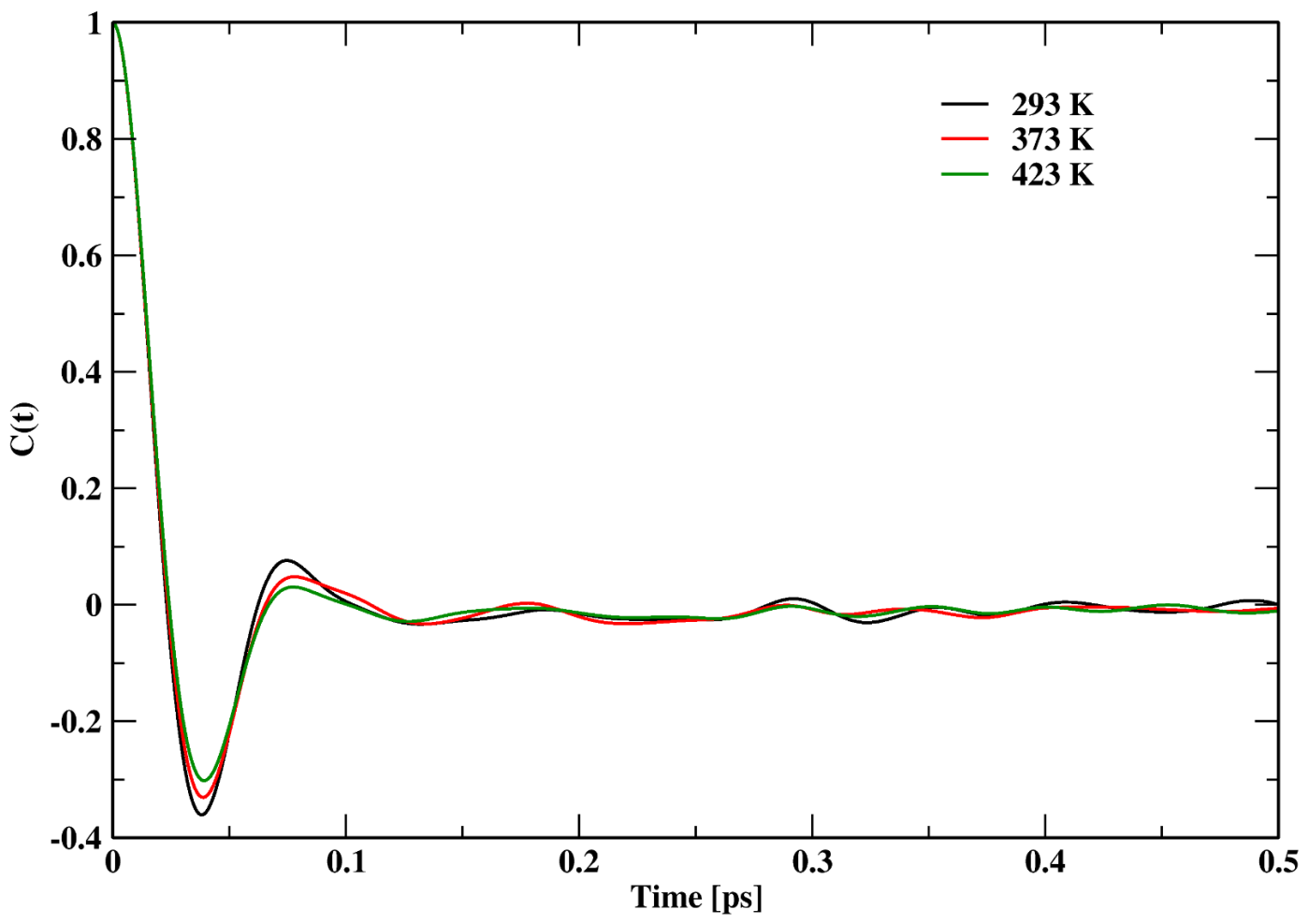

Figure S6. The figure shows the velocity autocorrelation function for lithium ions in LEC at 293,373 and $423 \mathrm{~K}$. The rattling motion is observed at all temperatures. 


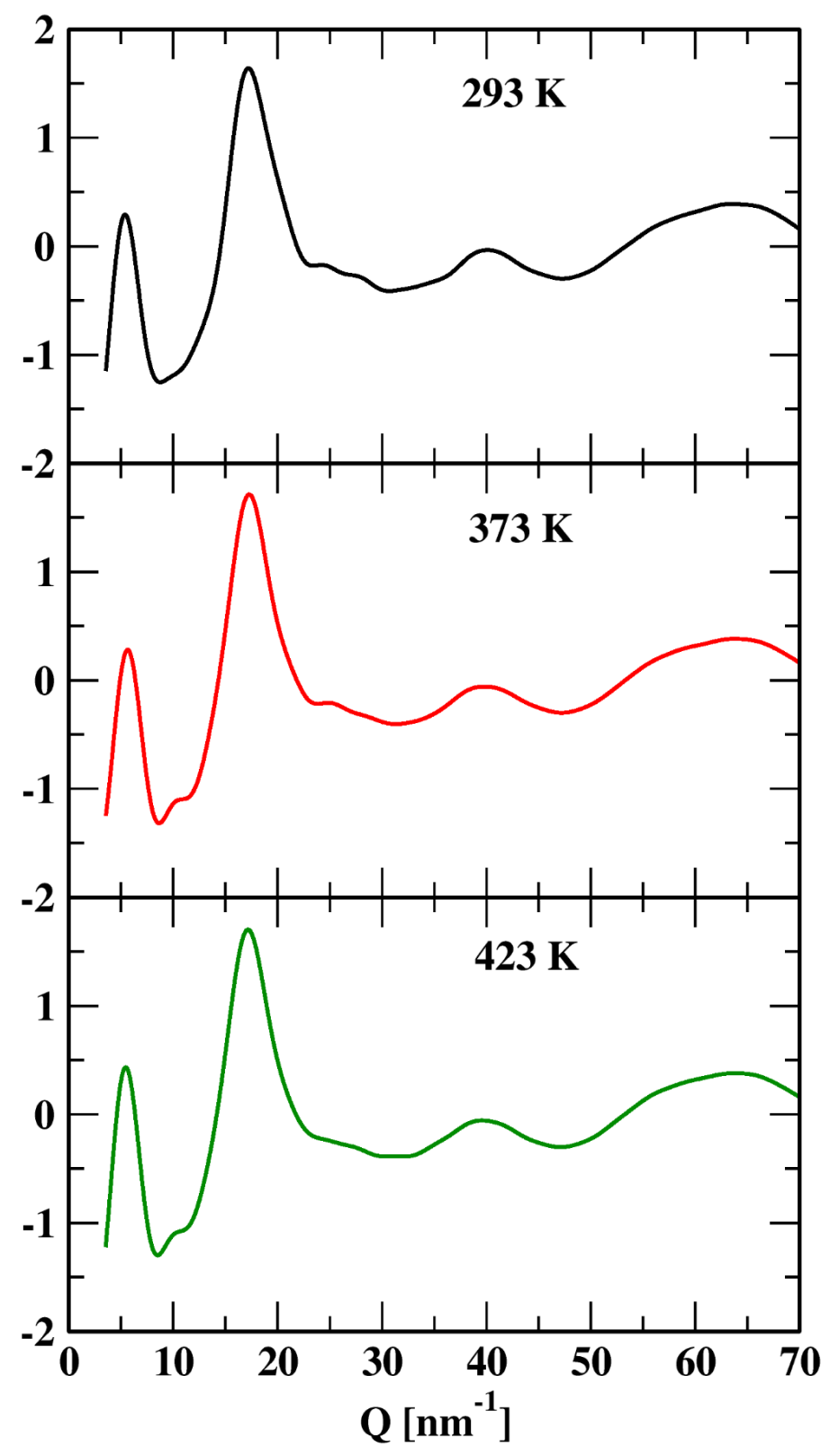

Figure S7. The variation of the Structure Factor plot for Lithium Methyl carbonate at three temperature values. Small shoulder peaks form at lower temperatures of 293 and 373K and at Q values greater than 20. 


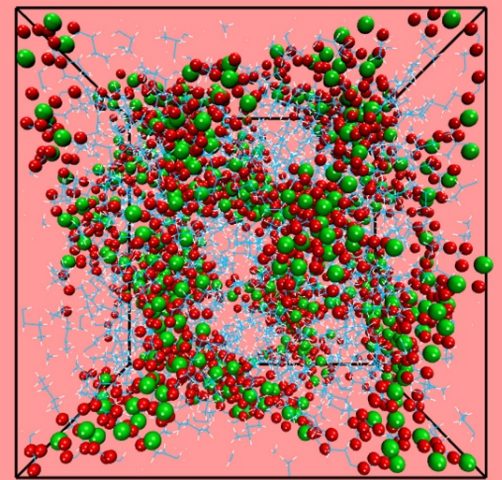

(a)

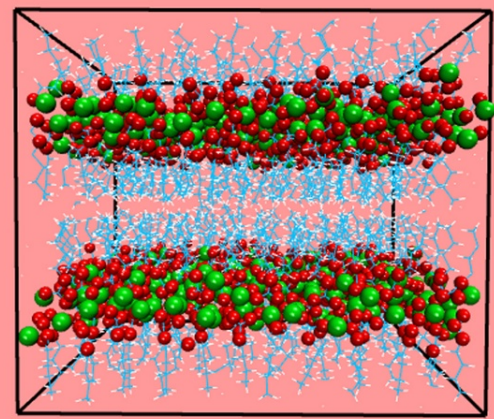

(c)

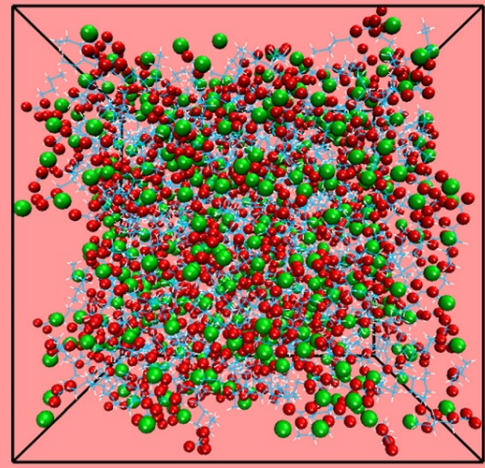

(e)

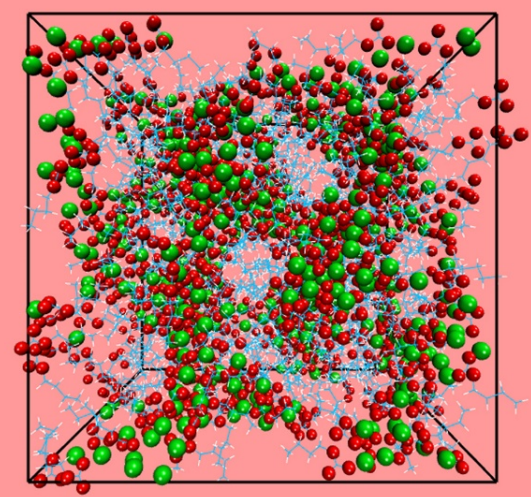

(b)

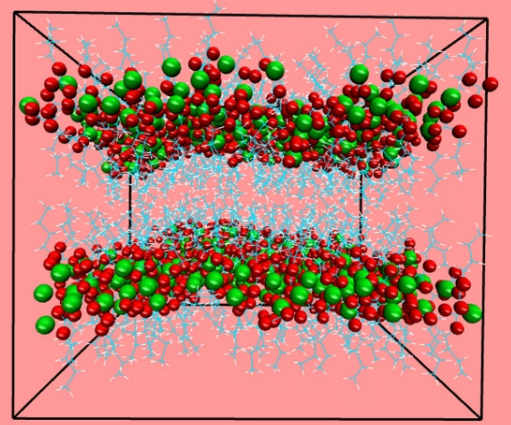

(d)

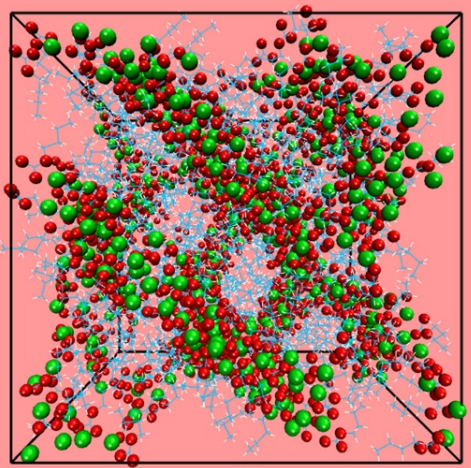

(f)

\section{Hydrogen Carbon}

Figure S8. The snapshots of the simulation box containing LBC species with the segregation of ions into layers can be seen. Illustrations (a), (b) and (e), (f) are the initial and final frames for the simulation at $293 \mathrm{~K}$ and $423 \mathrm{~K}$ respectively, starting from a completely random structure. Similarly (c), (d) are initial and final frames for the simulation of an ideal bilayer structure at 293K. 

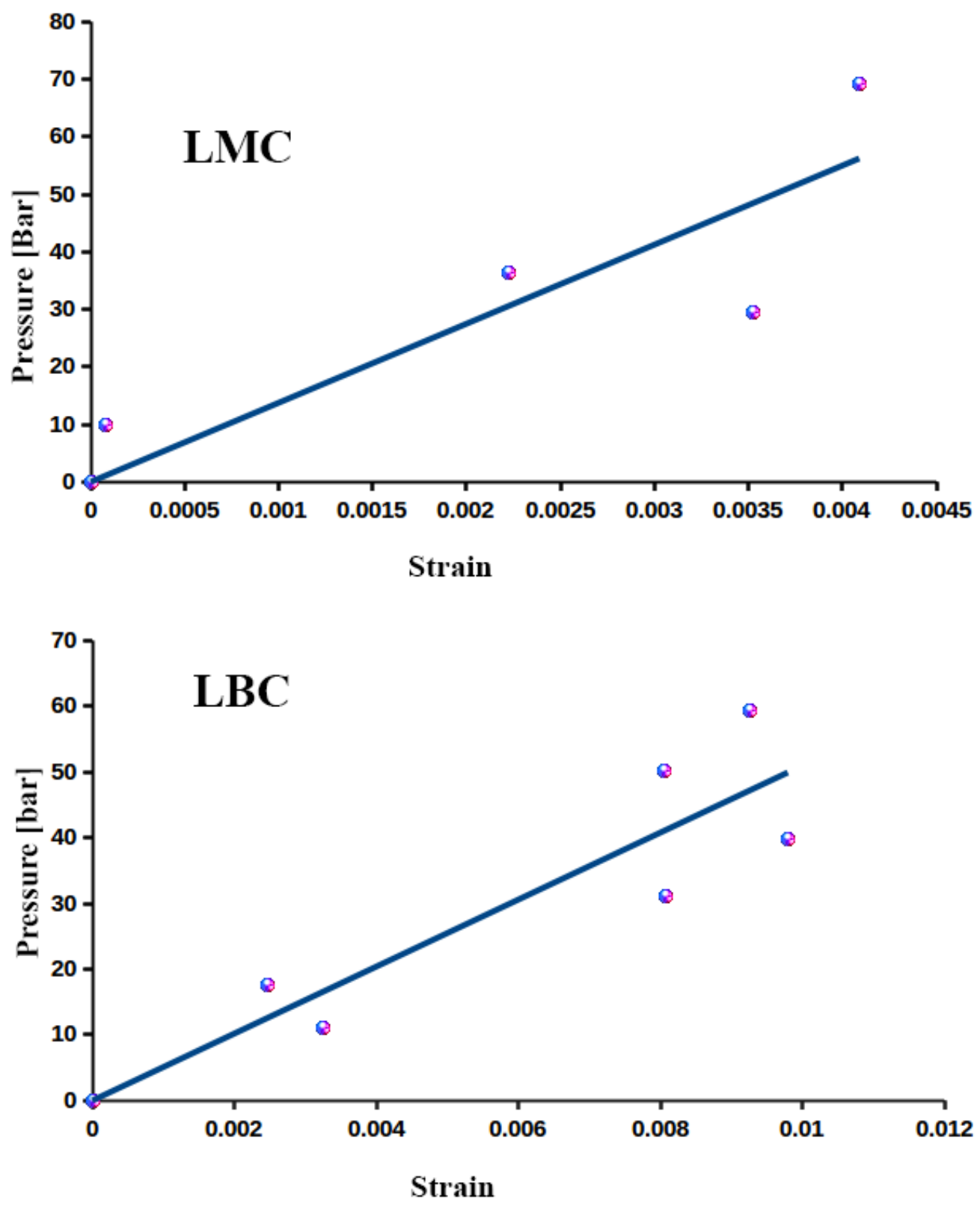

Figure 59. The Pressure-Strain plot for Lithium Methyl Carbonate and Lithium Butyl Carbonate at 293K. The pressure is relative to the average pressure obtained from the simulation at 1 bar. 


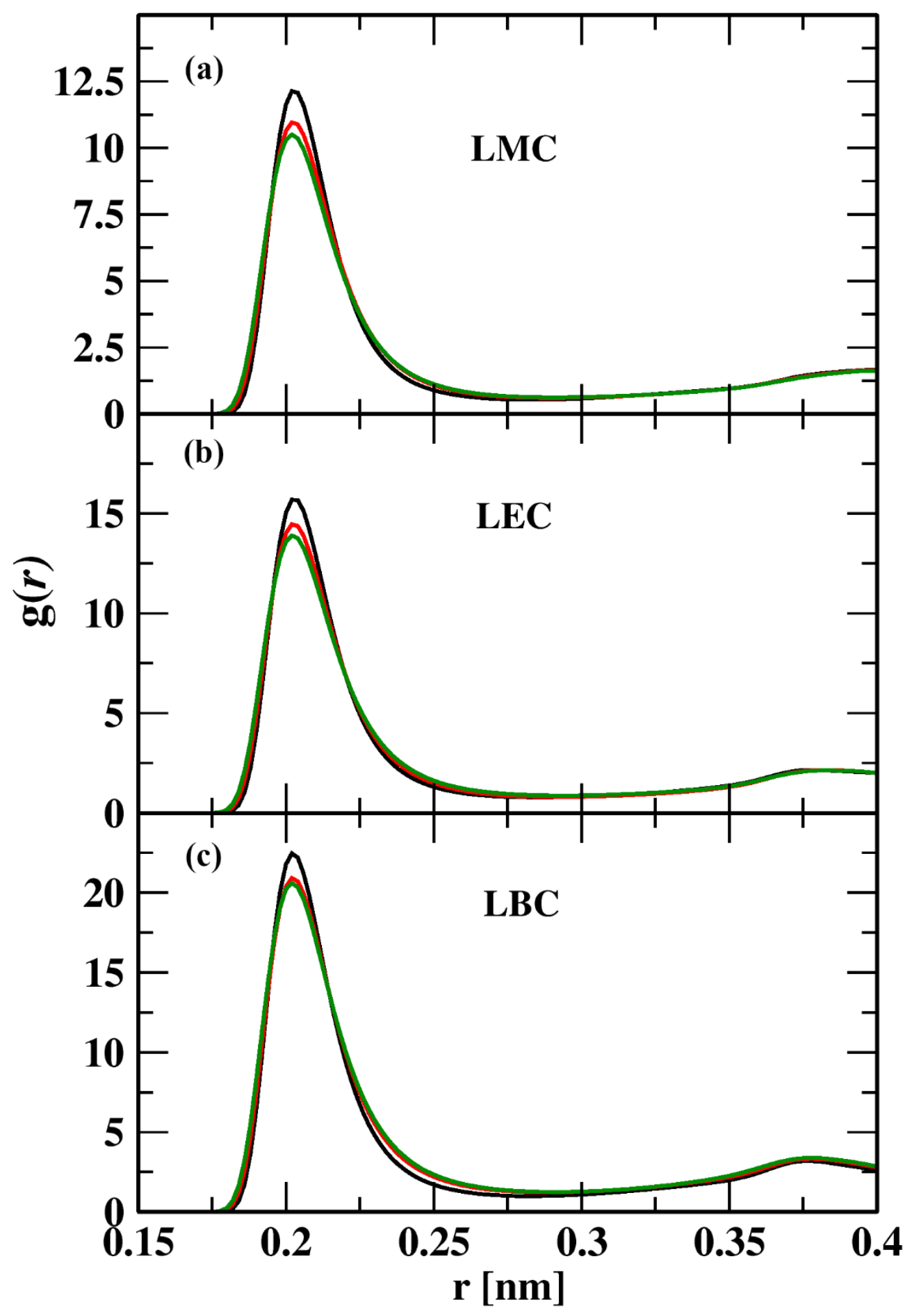

Figure S10. The radial distribution plots for LMC, LEC and LBC under the application of an Electric field of 4 Vnm-1. The peak maxima occur at an internuclear distance which is slightly greater than $0.2 \mathrm{~nm}$. 